\title{
TRIPLET STATES AND TRIPLET EXCITONS IN CHEMICALLY MIXED CRYSTALS OF ACRIDINE WITH ANTHRACENE
}

\author{
O. Morawski and J. Prochorow \\ Institute of Physics, Polish Academy of Sciences \\ Al. Lotników 32/46, 02-668 Warszawa, Poland
}

(Received May 9, 1995)

\begin{abstract}
Dedicated to Professors Krzysztof Pigon', Józef W. Rohleder and Zdzisław Ruziewicz on the occasion of their 70th birthdays*
\end{abstract}

Spectral and temporal characteristics and their temperature dependence for the long-lived (phosphorescence and delayed fluorescence) emission of chemically mixed crystals of acridine II (host) with anthracene (guest) were studied under conditions of various spectral resolutions and different modes of excitation. The energy of 0-0 transitions of exciton band and of trap states have been determined and the nature and energy scheme of the triplet states of crystals have been established. The complex arrangement of host molecules into two different pairs in the crystal structure of acridine II is revealed in the energetic "doublet" structure of the trap states for both, the shallow and the deep traps (of acridine and of anthracene origin, respectively). Migration of triplet excitation energy in these chemically mixed crystals is controlled predominantly by the triplet-triplet annihilation of excitons (either heterogeneous or homogeneous) almost in the whole temperature range. The formation of triplet excimers of acridine was definitely ruled out.

PACS numbers: $31.70 . \mathrm{Ks}, 33.50 . \mathrm{Dq}, 71.35 .+\mathrm{z}$

\section{Introduction}

Some time ago, the two-component, organic solid solutions with substitutional disorder, i.e. isotopically or chemically mixed molecular crystals seemed to be very attractive as the model systems for highly disordered systems (e.g. molecular glasses, polymers and liquids [1]). It has turned out, however, that in chemically

*Submitted on invitation of the Institute of Physical and Theoretical Chemistry, Technical University of Wrocław, Wrocław, Poland. 
mixed crystals, the most attractive factor, i.e. an anticipated easy-to-handle control of the degree of true substitutional (translational) disorder in the host crystal lattice, is connected with very severe geometrical and symmetry requirements [2]. Furthermore for most of the candidates, for the formation of chemically mixed crystals, a solid solubility limit very seldom exceeds a fraction of mol\%, thus limiting the range and degree of the controlled substitutional disorder and by the same token the generality of conclusions. On top of that, it has turned out in the course of experiments that even in such thermodynamically favourable cases like solid solution of anthracene and acridine where the host lattice can contain as much as tens of mol\% of the guest $[3,4]$, the analysis of experimental results may become very obscure (if not impossible at all) due to the fact that energy levels of host and guest and of different resonance aggregates are overlapping even at moderate guest concentrations, and both, the variety of aggregates and the overlap are strongly increasing with increasing guest concentration [5-7]. And although all these drawbacks and limitations have resulted in the shift of a centre of gravity of the research effort to isotopically mixed crystals, systematic studies of chemically mixed crystals (lightly and highly concentrated with the guest molecules) have provided some interesting results and concepts relevant to the exciton transport and trapping [8-11], formation of excited complexes and aggregation properties $[12,13]$ or aggregation-controlled molecular photophysics [7].

This work was aimed at identification of the triplet states of a new two-component solid solution of acridine (host) with anthracene (guest) which can form chemically mixed crystals. This system, in contrast to extensively studied "reversed" system of anthracene (host) with acridine (guest), has never been before a subject of optical studies, although chemically mixed crystals based on the acridine host lattice should be very interesting from the point of view of excitation energy transfer, due to the variety of crystalline modifications of a neat acridine crystal and the pairwise arrangement of the molecules in the lattice $[14,15]$ which may dominate in photophysics and kinetics of excited states and overwhelm the resonance (guest-guest) interactions. With this motivation we have extended our previous interest in chemically mixed crystals of anthracene (host) with acridine (guest) to the crystals under consideration.

The details relating to the electronic singlet states, traps for singlet excitons as well as the verification of the formation of singlet excimers of acridine in chemically mixed crystals of acridine II with anthracene, have been presented in our recent paper [16] together with some preliminary results of low-resolution optical studies of long-lived emission spectra. We recall that in all studied crystals [16]: (i) the energy of the bottom of singlet excitonic band is $25450 \mathrm{~cm}^{-1}$ (it is of acridine $S_{1}\left(\pi, \pi^{*}\right)$ origin); (ii) the only traps for singlet excitons are of anthracene origin, with energy of $c a .24000 \mathrm{~cm}^{-1}$ but the efficiency of trapping is small even at low temperatures; (iii) singlet excitons from the singlet excitonic band are decaying predominantly via the process of formation of excimers (either in $\mathrm{A}$ or $\mathrm{B}$ pairs - see Sec. 3 below), the energy of excimer state is $c a .21000 \mathrm{~cm}^{-1}$ and its radiative decay time is of $85 \mathrm{~ns}$. These findings were in general agreement with the conclusions of earlier spectroscopic experiments performed for the neat acridine II crystals $[15,17,18]$. For the first time, however, we have observed the phosphores- 
cence emission of the crystal in acridine II crystallographic form. This observation supplied us with a tool, which together with high-resolution laser excitation, could have been used for investigations of the triplet states and triplet excitons and for verification of the concepts of a triplet excimer as well as for insight into the photophysical mechanism of deactivation of excited molecular and/or excimer singlet states. The results of detailed studies of the phosphorescence emission and its kinetics are summarized and discussed in this paper.

\section{Experimental}

Anthracene and acridine were purified by vacuum sublimation and zone-refining. Crystals of $0.02,0.1$ and $1.0 \% \mathrm{M} / \mathrm{M}$ anthracene in acridine host were grown by sublimation [4]. With the use of the standard powder diffractometry technique a crystal structure of the host lattice in mixed crystals was found to coincide with the crystal structure of acridine II.

A photon-counting technique was used for recording the emission spectra. Several modes of excitations have been employed, i.e. 365 and $405 \mathrm{~nm}$ lines isolated from a mercury IIBO 200 lamp; for selective excitation within the $395-415 \mathrm{~nm}$ range - a dye laser (Lambda Physik FL3001) pumped by $\mathrm{XeCl}$ excimer laser (Lambda Physik LPX105); for excitation spectra - the above laser setup equipped with KDP frequency doubler (FL 30 SHG crystal) tuned within 605-660 nm range with a linewidth of $0.4 \mathrm{~cm}^{-1}$ or $0.08 \mathrm{~cm}^{-1}$ (with FL 82 Fabry-Perot etalon set).

All details of the experimental setup, including optical helium cryostat and the electronics as well as the techniques of decays measurements, have been published previously [19].

\section{Results and discussion}

At least five different crystalline modifications of acridine have been reported up to now (see [15] and the references therein). In the present study of chemically mixed crystals, a crystalline form of acridine II (monoclinic, with 8 molecules in the unit cell and the space group $P 2_{1} / a$ ) serves as the host lattice for anthracene molecules. The crystallographic structure of acridine II, with two molecules per asymmetric unit, has a complex arrangement of molecules. The packing units, each containing two molecules, are not all equivalent as the molecules are arranged in two types of antiparallel pairs about the centre of symmetry (designated as A and B pairs, respectively). The separation distance between molecules in orientation $A$ is $0.349 \mathrm{~nm}$ and in normal projection they are not exactly overlapped but are staggered. In B orientation, the separation distance is $0.361 \mathrm{~nm}$ and in normal projection there is only an overlap of the terminal aromatic rings of the two acridine molecules [14].

In general, such a crystallographic structure of the host crystal should provide more sites for substitution and larger variety of local environments for guest molecule than a "reversed" mixed crystal of anthracene (host) with acridine (guest). However, it has been shown long ago that in the acridine II structure anthracene is not able to pack as closely as acridine molecules [3] and this fact strongly limits an accessible concentration range of guest molecules in these chemically mixed 
crystals. Hence, our investigations have been limited to three mixed crystals of acridine with anthracene concentrations $0.02,0.1$ and $1.0 \% \mathrm{M} / \mathrm{M}$. In all these crystals phosphorescence emission was observed and its detailed features and the temperature dependence are discussed in the following.

\subsection{Phosphorescence at low temperatures}

Figure 1 illustrates the long-lived emission spectra of chemically mixed crystals of acridine and anthracene, at $1.7 \mathrm{~K}$, for different concentrations of anthracene in the acridine II host lattice. This emission is composed of a broad, and structureless delayed fluorescence band, spread out in $21000-17000 \mathrm{~cm}^{-1}$ spectral range, which is identical in its shape and spectral position with the spectrum of prompt fluorescence of the crystals under consideration [16]. On the low-energy side of delayed fluorescence band (below $16000 \mathrm{~cm}^{-1}$ ), a phosphorescence emission is observed. The phosphorescence spectrum is composed of very sharp and fairly well resolved bands. The energy of maximum of the highest-energy band in phosphorescence spectrum (a presumable 0-0 transition of phosphorescence emission) is ca. $15691 \mathrm{~cm}^{-1}$. At $1.7 \mathrm{~K}$, the decay time of phosphorescence monitored at this band is $33 \mathrm{~ms}$. Both, the energy of this phosphorescence band and its decay time can be compared to the energy of 0-0 transition $\left(15813 \mathrm{~cm}^{-1}\right)$ and the decay time of phosphorescence (34 ms) observed for acridine isolated (at low concentrations) in the crystalline host lattice of 2,3-dimethylnaphthalene [20]. And such a comparison would suggest a rather safe assignment of the phosphorescence spectrum observed in these mixed crystals as the phosphorescence of acridine.

However some other characteristics of phosphorescence emission indicate that such an assignment would be an oversimplified one. First of all, it is seen in Fig. 1 that intensity distribution among different subbands of the phosphorescence spectrum is different for different concentrations of anthracene in the acridine host lattice. In mixed crystals with 0.02 and $0.1 \%$ of anthracene the $0-0$ band at $15691 \mathrm{~cm}^{-1}$ is the most intense one (Fig. 1a and 1b), but in the crystal with the highest concentration $(1.0 \%)$ of anthracene its intensity is strongly diminished and the band at $14829 \mathrm{~cm}^{-1}$ is the most intense one (Fig. 1c). Another, very distinct observation related to the changes of the intensity distribution within the subbands of phosphorescence spectrum is given in Fig. 2, which shows that even for a fixed concentration of anthracene, the relative intensities are dependent on the wavelength of excitation: If excitation is carried out via singlet excitonic band (27472 $\mathrm{cm}^{-1}$ excitation) the $0-0$ band of phosphorescence of acridine, at $15690 \mathrm{~cm}^{-1}$, is the most intense one. When the excitation is tuned below the singlet excitonic band, directly to the singlet traps $\left(24619 \mathrm{~cm}^{-1}\right.$ excitation) the intensity of the $0-0$ band (and of some other subbands) is decreasing, while the intensity of the band located at $14829 \mathrm{~cm}^{-1}$ is strongly enhanced.

A more detailed analysis of the vibrational pattern of the phosphorescence spectrum enables the identification (with accuracy of $\pm 5 \mathrm{~cm}^{-1}$ ) of such vibrational frequencies as 225, 400,620,1276, 1410 and $1569 \mathrm{~cm}^{-1}$ which are characteristic of acridine (and have been all identified in phosphorescence spectrum of acridine embedded as a dopant into the crystal of fluorene [21]). Thus we notice that a $c a .860 \mathrm{~cm}^{-1}$ shift between the most intense bands under considera- 

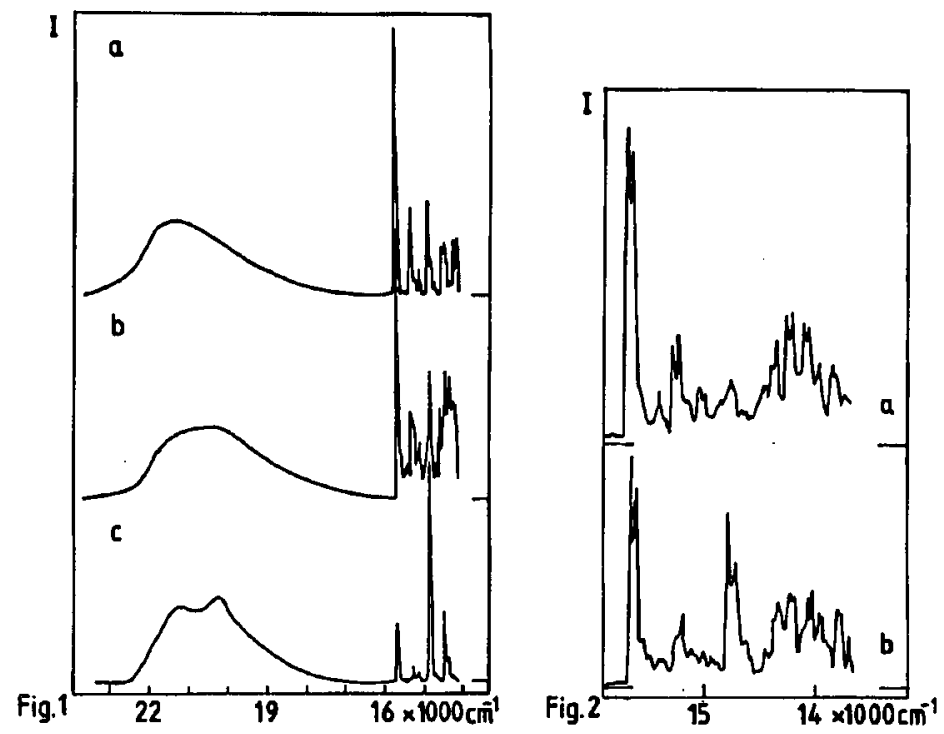

Fig. 1. Delayed fluorescence and phosphorescence spectra, at $1.7 \mathrm{~K}$, of chemically mixed crystals of acridine II with anthracene for different concentrations of anthracene, with excitation to the singlet excitonic band $\left(27472 \mathrm{~cm}^{-1}\right)$. Concentrations of anthracene: (a) $-0.02,(\mathrm{~b})-0.1$, (c) $-1.0 \mathrm{~mol} \%$.

Fig. 2. Phosphorescence spectra of the chemically mixed crystal of acridine II with $0.02 \mathrm{~mol} \%$ of anthracene recorded at $4.2 \mathrm{~K}$ for different excitations. Excitation energies: $27472 \mathrm{~cm}^{-1}$ (a) and $24619 \mathrm{~cm}^{-1}$ (b).

tion (15691-14829 $\mathrm{cm}^{-1}$ ) is not connected with any vibrational mode of acridine molecule and hence the band at $14829 \mathrm{~cm}^{-1}$ must be related to the emitting species (site) different from acridine molecule. This conclusion is further supported by the fact that the decay time of phosphorescence, monitored at this band is $40 \mathrm{~ms}$, at $1.7 \mathrm{~K}$, which is different from $34 \mathrm{~ms}$ observed for $15691 \mathrm{~cm}^{-1}$ band. In view of these observations the $14829 \mathrm{~cm}^{-1}$ band can be identified as that of anthracene origin. For comparison, the 0-0 band of anthracene phosphorescence in the host lattice of biphenyl crystal is located at $14738 \mathrm{~cm}^{-1}$ [22] and its decay time is $42 \mathrm{~ns}$ [23] (in phenazine crystal its position is $14686 \mathrm{~cm}^{-1}$ [24]).

Hence, we arrive at the conclusion that the phosphorescence of mixed crystals of acridine with anthracene consists of two overlapping spectra: the high-energy phosphorescence spectrum with the $0-0$ band at $15691 \mathrm{~cm}^{-1}$ (and the decay time of $34 \mathrm{~ms}$ ) which is of acridine origin and the low-energy spectrum with the $0-0$ band at $14829 \mathrm{~cm}^{-1}$ (and the decay time of $40 \mathrm{~ns}$ ) which is of anthracene origin. Each of these two spectra can dominate in the total phosphorescence of mixed crystals, depending on either concentration of anthracene (cf. Fig. 1) and the mode of excitation (cf. Fig. 2), or the temperature range (see the discussion in Sec. 3.2). However, the general pattern of the phosphorescence spectrum in the mixed crystals under study clearly shows that the phosphorescence originates from 
two kinds of triplet traps of different depth and origin. There are shallow traps of acridine origin and much deeper traps (by $c a .870 \mathrm{~cm}^{-1}$ ) of anthracene origin, which might have been expected in view of the energy difference between the triplet states of both molecules. As we have shown earlier [16] singlet traps in the mixed crystals of acridine with anthracene are formed exclusively on anthracene molecules (and those can be traced in both, the fluorescence and absorption spectra of the crystal) and this conclusion is in general agreement with the observed enhancement of anthracene phosphorescence upon excitation tuned to singlet traps, below the singlet excitonic band (cf. Fig. 2). The shallow acridine traps are populated mostly via excitation to the singlet excitonic band which, as we have demonstrated earlier, serves as a precursor for the formation of acridine excimer in the crystal [16]. As the observed acridine phosphorescence spectrum has a very sharp and clear-cut molecular-like character in the crystal and as even at low temperatures its intensity is almost three orders of magnitude smaller than the intensity of excimer-like fluorescence, it seems highly improbable that acridine traps can be populated with the participation of excimer states. Thus, we must conclude that the most probable mechanism for population of acridine triplet traps is that connected. with direct intersystem crossing from the singlet excitonic band to the triplet excitonic band of the host crystal from where shallow acridine traps are being populated. We also stress that contrary to confirmed formation of singlet acridine excimers, the postulated formation of triplet excimers $[15,18,19]$ in the acridine II crystal can definitely be ruled out.

An inspection of even relatively low-resolution spectra given in Fig. 2, is revealing that the $0-0$ bands of both, the acridine and anthracene phosphorescence spectra (as well as some other subbands in both spectra) exhibit a composite structure. The high-resolution phosphorescence spectra clearly show their "doublet" structure, as illustrated in Fig. 3 for the acridine part of the phosphorescence spectrum. The components of the "doublet" have energies of 15691 and $15649 \mathrm{~cm}^{-1}$, respectively. The $42 \mathrm{~cm}^{-1}$ separation between the "doublet" components is characteristic of all the other bands in the spectrum (and the same separation characterizes the "doublet" structure in the anthracene phosphorescence spectrum, for which the "doublet" components of the 0-0 band are located at 14829 and $14787 \mathrm{~cm}^{-1}$, respectively). Such a "doublet" character of the phosphorescence from both kinds of traps (of acridine and of anthracene origin) can only be accounted for if one assumes that in both cases there are two different sites for trapping molecules. Since the separation between the "doublet" components is practically the same for the sets of shallow (acridine) traps and deep (anthracene) traps, the trapping sites must be connected with a specific structure of the acridine II crystal lattice and must reflect the differences in the "local" situation of the triplet states of trapping molecules within the $\mathrm{A}$ and $\mathrm{B}$ pairs (see beginning of Sec. 3). In the zeroth-order approximation, the energy of the triplet state of either the host molecule (acridine) or substitutionally replacing it anthracene molecule would be controlled by the distance between the components of the pair, which is the same for acridine and anthracene molecule in any given pair (provided, in these chemically mixed crystals, a true substitutional replacement takes place) but is different in A and in B pairs. Our present results do not allow for the 




Fig. 3. Phosphorescence of shallow (acridine) traps in the chemically mixed crystal of acridine II with $0.02 \mathrm{~mol} \%$ of anthracene at different temperatures (as indicated). Excitation energy $27472 \mathrm{~cm}^{-1}$.

precise determination of the local structure of the shallow and deep triplet traps and/or trapping sites and for instance "an anti-parallel arrangement" of acridine molecules within the A and B pairs as a possible triplet trap cannot be excluded. Nevertheless, the identification of two different sets of triplet traps, each of which is divided into two different subsets (sites) of traps is beyond any doubt. In order to determine the energy of the triplet excitonic band of the acridine II host crystal we have measured phosphorescence excitation spectra with the use of a narrow-band tunable dye laser. The excitation spectrum of acridine phosphorescence (in the $0-0$ region) is presented in Fig. 4. The two most intense lines at $15821.0 \mathrm{~cm}^{-1}$ and $15752.9 \mathrm{~cm}^{-1}$ can be ascribed to the two Davydov components of the host triplet excitonic band. Other lines of much smaller intensity form two progressions with identical shifts $\left(255,400,440 \mathrm{~cm}^{-1}\right.$, etc.) from both Davydov components. At still higher resolution the shape of both Davydov components (cf. Fig. 5) seems to suggest a presence of more "finer splitting" which could not be further resolved under present experimental conditions. This should be investigated under appropriate resolving power, especially as it should be born in mind that although the crystal structure of acridine II is arranged pairwise (which probably dominates all the existing couplings and interactions), still, however, there are 8 molecules in the elementary unit cell of the crystal, what may result in the complicated pattern of the Davydov splitting [25].

The above-presented results and discussion can be summarized in the energy scheme of the triplet states in the chemically mixed crystal of acridine with anthracene given in Fig. 6. From this we can determinate the depths of triplet traps observed in these crystals. These depths (relative to the lower Davydov component) are equal to 62 and $1.04 \mathrm{~cm}^{-1}$ for the set of acridine traps and 924 and $966 \mathrm{~cm}^{-1}$ for the set of deep anthracene traps, respectively. The reliability of these values and validity of some conclusions formulated in the preceding can be verified by investigations of the kinetics of triplet excitons. 



Fig. 4. Excitation spectrum of phosphorescence (in 0-0 region) of shallow (acridine) traps, at $4.2 \mathrm{~K}$, in the chemically mixed crystal of acridine II with $0.02 \mathrm{~mol} \%$ of anthracene.

Fig. 5. The shape of low and high Davydov components of Fig. 4 at high resolution.
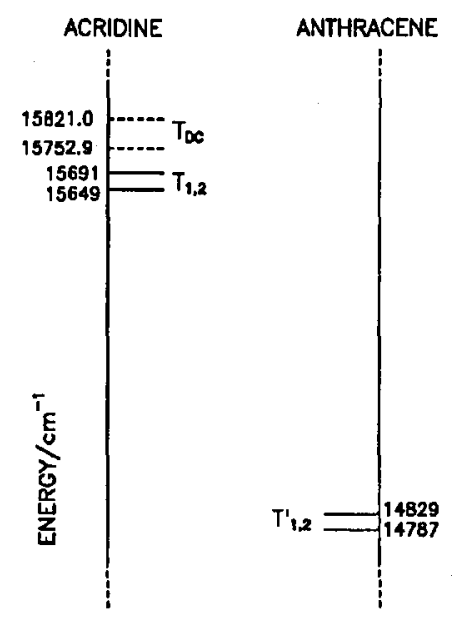

Fig. 6. Scheme of triplet energy levels of the chemically mixed crystal of acridine II with anthracene. The exciton bands of the host (acridine II) are represented by their Davydov components $\left(T_{\mathrm{DC}}\right)$. Two sets of traps of different origin are depicted as: $T_{1,2}$ - shallow traps of acridine origin and $T_{1,2}^{\prime}-$ deep traps of anthracene origin (see text for the details).

\subsection{Temperature dependence of phosphorescence and the kinelics of triplet excitons}

The quoted earlier Fig. 3 illustrates also the temperature dependence of the "doublet" components of phosphorescence spectrum originating from acridine traps. With an increase in temperature the high-energy component is loosing its 
intensity much faster than the low-energy component and above $10 \mathrm{~K}$ only this latter one can be still observed. Its intensity is quickly decreasing with temperature and above $15 \mathrm{~K}$ the phosphorescence of acridine is no longer existing in the phosphorescence spectrum of the mixed crystal. What is left is the anthracene part of phosphorescence spectrum of the crystal, which (after some initial increase in intensity in the temperature range of $15-20 \mathrm{~K}$ ) is also loosing its intensity with increasing temperature and eventually ceases to exist in the temperature range of 80-100 K. The temperature dependence of the whole phosphorescence spectrum, together with the temperature changes of delayed fluorescence spectrum are shown in Fig. 7 for low-resolution spectra. The temperature dependence of the integral intensity of delayed fluorescence although somewhat more complicated than that observed for phosphorescence, reveals four characteristic regions of temperature for which its behaviour is clearly displayed. It starts to grow at low temperatures (above $2 \mathrm{~K}$ ), reaches the maximum (at $\sim 15 \mathrm{~K}$ ) and after a slight drop remains constant in the range of medium temperatures (up to $\sim 60 \mathrm{~K}$ ) and then starts once again to increase sharply and reaches its second maximum (at $\sim 100 \mathrm{~K}$ ). Above $100 \mathrm{~K}$ the intensity of delayed fluorescence is slowly but continuously decreasing with increasing temperature. All these temperature changes of intensity of delayed fluorescence and trap phosphorescence of acridine and anthracene are accompanied by temperature changes of their decay times (cf. Fig. 8).

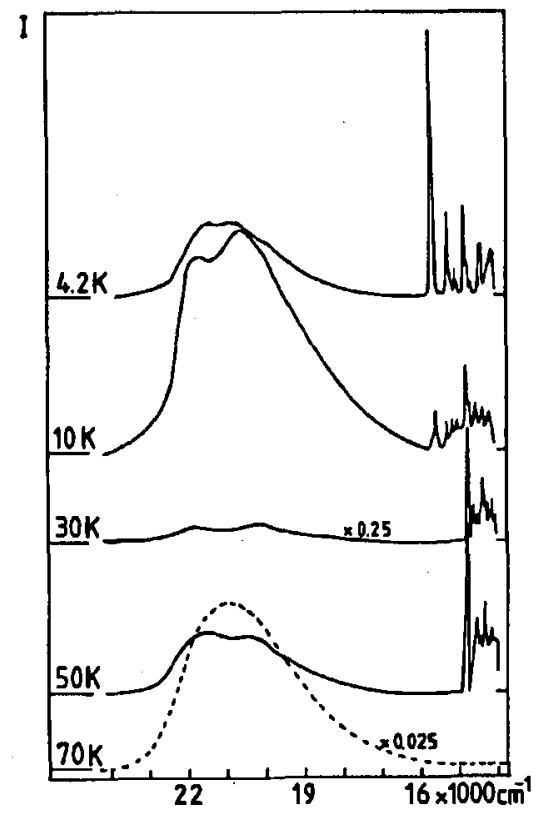

Fig. 7. Delayed fluorescence and phosphorescence spectra of the chemically mixed crystal of acridine II with $0.02 \mathrm{~mol} \%$ of anthracene at different temperatures (as indicated). Excitation energy $27472 \mathrm{~cm}^{-1}$. 


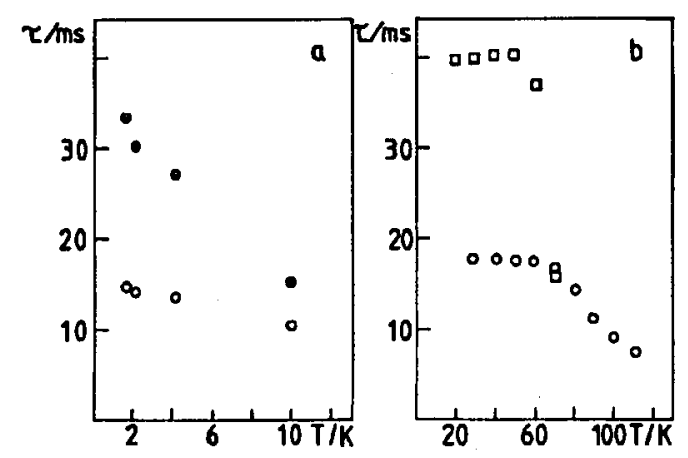

Fig. 8. Decay times of delayed fluorescence (o) and phosphorescence: of acridine traps $(\bullet)$ in 1.7-10 K temperature range (a) and of anthracene traps ( $\diamond)$ in $20-110 \mathrm{~K}$ temperature range (b) in the chemically mixed crystal of acridine II with $0.02 \mathrm{~mol} \%$ of anthracene. Excitation at $27472 \mathrm{~cm}^{-1}$. Decays of each phosphorescence monitored at its 0-0 range, excitation with $27472 \mathrm{~cm}^{-1}$.

These observations of temperature changes of delayed fluorescence are typical for delayed fluorescence of triplet-triplet annihilation origin (P-type delayed fluorescence) in the almost whole range of investigated temperatures. At deep temperatures $(1.7-2.0 \mathrm{~K})$ most of the excitons are trapped, which results in intense phosphorescence (mostly from shallow acridine traps) and relatively low-intensity delayed fluorescence which must be of heterogeneous triplet-triplet annihilation between mobile triplet excitons in the excitonic band and trapped excitons. This is also confirmed by the fact that the decay time of delayed fluorescence at $1.7 \mathrm{~K}$ is $15 \mathrm{~ms}$ (cf. Fig. 8) which is almost twice as short as the decay time of acridine phosphorescence (34 ms) - a feature typical for the emission originating from the annihilative events. When the temperature is increased, excitons from shallow acridine traps are thermally activated to the triplet excitonic band, the intensity of phosphorescence drops (c.f. Figs. 3 and 7) and its decay time is getting shorter. At the same time (in $2-15 \mathrm{~K}$ range) the intensity of delayed fluorescence is quickly increasing (see above). In this temperature range the delayed fluorescence is due to the homogeneous annihilation of mobile excitons (although annihilation of mobile excitons and excitons trapped on deep anthracene traps can also contribute, especially in the mixed crystals with higher concentration of anthracene). Some fraction of all excitons which are thermally activated from acridine traps is also being repumped to deep anthracene traps, as can be inferred from the above-mentioned increase in intensity of anthracene phosphorescence at $15-20 \mathrm{~K}$ range. We notice that at $20 \mathrm{~K}$ and up to $\sim 50 \mathrm{~K}$, the decay time of delayed fluorescence (cf. Fig. 8) is $18 \mathrm{~ms}$ (although it has fallen down to $\sim 10 \mathrm{~ms}$ at $15 \mathrm{~K}$ ), and is almost twice as short as the decay time of anthracene phosphorescence $(40 \mathrm{~ms})$. This indicates that after depopulation of shallow acridine traps, the heterogeneous annihilation of mobile excitons and excitons trapped on deep anthracene traps becomes important. When the temperature is increased above $\sim 40-50 \mathrm{~K}$, the intensity of anthracene phosphorescence and its decay time start to decrease quickly and this 
means that the thermal activation of deep triplet traps becomes more and more effective. Above $\sim 80 \mathrm{~K}$, deep triplet traps are for all practical purposes empty (phosphorescence can no longer be observed) and the delayed fluorescence which is still observed is due to the homogeneous triplet-triplet annihilation of mobile excitons. A continuous decrease in its intensity and in decay time observed for higher temperatures is due to scattering of mobile excitons travelling trough the crystal lattice, as the scattering increases with increasing temperature of the crystal.

It comes, from the above-outlined, qualitative description that there are two temperature ranges where the intensity of delayed fluorescence $\left(I_{\mathrm{df}}\right)$ is governed and controlled by the effective thermal activation of trapped triplet excitons to the triplet excitonic band, i.e.:

$$
I_{\mathrm{df}} \propto n_{\mathrm{p}}^{2} \exp (-\Delta E / k T),
$$

where $n_{\mathrm{p}}$ is the population of the trap, $\Delta E$ is the activation energy for the depopulation process, $k$ - Boltzmann constant. Since the phosphorescence intensity $\left(I_{\mathrm{ph}}\right)$ is proportional to the population of trap $n_{\mathrm{p}}$, an Arrhenius type of plot of $\ln \left(I_{\mathrm{df}} / I_{\mathrm{ph}}^{2}\right)$ versus reciprocal temperature $(1 / T)$ should bring about an activation energy, $\Delta E$, for the process of thermal depopulation of given traps. In Fig. 9 such an Arrhenius plot which relates to thermal depopulation of deep anthracene traps (50-90 K temperature range) is given. The activation energy for this process obtained from the plot in Fig. 9 is equal to $940 \pm 60 \mathrm{~cm}^{-1}$. If we compare this value to the average depth of anthracene traps which were obtained from spectroscopic data (see Sec. 3.1) and which are 922 and $961 \mathrm{~cm}^{-1}$, we see that these values are in excellent agreement. An analogous procedure applied for shallow acridine traps (for 2-10 K temperature range) yields the activation energy of $60 \pm 6 \mathrm{~cm}^{-1}$ which is also in good agreement with $60 \mathrm{~cm}^{-1}$ determined from spectroscopic data (see Sec. 2.2) as the depth of higher-energy subset of acridine triplet traps. These activation energies show that the proposed photokinetic scheme not only well ac-

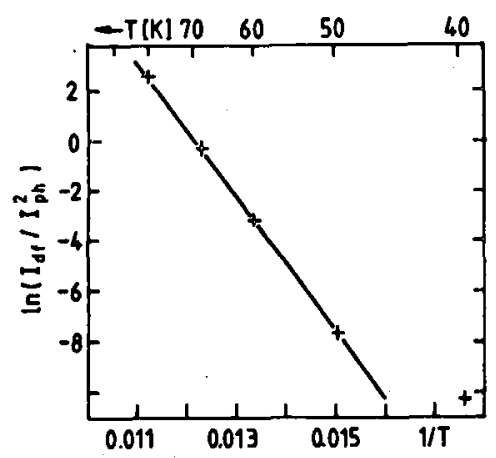

Fig. 9. An Arrhenius-type plot of the intensities of delayed fluorescence and phosphorescence for thermally activated depopulation of deep (anthracene traps) in 50-90 K temperature range (see text for the details). 
counts for the observed behaviour of the long-lived emission of these chemically mixed crystals (although this scheme would necessitate some minor corrections for some of the temperature ranges in the case of crystals which contain higher concentrations of anthracene ( 0.1 and especially $1.0 \%)$ ), but also is self-consistent from the quantitative point of view.

\subsection{Concluding remarks}

Determination of the nature and the energy scheme of the triplet states in the chemically mixed crystals of acridine II with anthracene has revealed that the triplet traps of both types (of acridine and anthracene origin) are within each type very distinctly segregated into two groups (of energy). We believe that this is a clear demonstration of a very selective role of the structure of the host crystal lattice, which in the case of acridine II with two molecules per asymmetric unit offers two distinctly different local environments (sites) for anthracene guest molecule. It is also a proof that with this moderate concentration of guest molecule a true substitutional replacement of host by guest takes place.

A complete lack of any traces of the formation of triplet excimers of acridine may be considered as a piece of evidence which would rule out the nonradiative singlet-triplet intersystem crossing (from excimer singlet state) as a process controlling the decay of acridine singlet excimers, thus leaving a thermally activated internal conversion as the main nonradiative mode of excimer's decay. Still, however, population of the triplet states (traps), though of very low efficiency, takes place in these crystals and an understanding of how the triplet states (traps) are populated is probably of great importance for photophysics of acridine (not only in the crystalline phase). In order to solve this problem one has to probe the dynamics of the formation and the decay of singlet excimer of acridine with the picosecond time-resolved emission studies and this is now in the course of investigations for acridine II and its chemically mixed crystals in this laboratory.

\section{Acknowledgments}

We are deeply grateful to Dr. R. Radomski (Technical University of Wrocław) for supplying us with many samples of chemically mixed crystals, among them, with those particular which were used in this work. We also wish to thank him for stimulating inspiration and many valuable suggestions on various aspects of chemically mixed molecular crystals, from which we have benefited over the years. We also acknowledge the financial support of this research from the State Committee for Scientific Research (Republic of Poland) under the Project nr 223339203.

\section{References}

[1] R. Kopelman, in: Spectroscopy and Excitation Dynamics of Condensed Molecular Systems, Eds. V.M. Agranovich, R.M. Hochstrasser, North-Holland, Amsterdam 1983, pp. 139-184.

[2] A.I. Kitaigorodski, Mixed Crystals, Springer, Berlin 1983.

[3] R.M. Miasnikowa, A.I. Kitaigorodski, Crystallographia 3, 160 (1958). 
[4] M. Radomska, R. Radomski, K. Pigoń, Mol. Cryst. Liq. Cryst. 18, 75 (1972).

[5] B. Kozankiewicz, O. Morawski, J. Prochorow, R. Radomski, Mater. Sci. XIII, 129 (1987).

[6] O. Morawski, B. Kozankiewicz, J. Prochorow, R. Radomski, Chem. Phys. Lett. 150, 307 (1988).

[7] O. Morawski, B. Kozankiewicz, J. Prochorow, R. Radomski, Chem. Phys. Lett. 173, $412(1990)$.

[8] S.T. Gentry, R. Kopelman, J. Phys. Chem. 88, 3170 (1984).

[9] J. Grimm, T. Kirski, C. von Borczyskowski, Chem. Phys. Lett. 128, 569 (1986).

[10] S.A. Gilbert, T. Kirski, H. Brenner, C. von Borczyskowski, Chem. Phys. Lett. 178, 379 (1991).

[11] C.E. Mooney, S.J. Duray, D.D. Smith, Chem. Phys. Lelt. 178, 419 (1991).

[12] J. Grimm, C. von Borczyskowski, Chem. Phys. Lett. 174, 294 (1990).

[13] A. Kitzinger, H. Port, Chem. Phys. Lett. 184, 11 (1991).

[14] D.C. Philips, F.R. Ahmed, W.H. Barnes, Acla Crystallogr. 13, 365 (1960).

[15] J.O. Williams, B.P. Clarke, J. Chem. Soc. Faraday Trans. II 73, 514 (1977).

[16] O. Morawski, J. Prochorow, R. Radomski, Mol. Cryst. Liq. Cryst. 230, 173 (1993).

[17] B.P. Clarke, J.M. Thomas, J.O. Williams, Chem. Phys. Lett. 35, 251 (1975).

[18] J.O. Williams, B.P. Clarke, M.J. Shaw, Chem. Phys. Lett. 39, 142 (1976).

[19] B. Kozankiewicz, J. Prochorow, Mol. Cryst. Liq. Cryst. 148, 93 (1987).

[20] O. Morawski, J. Prochorow, Chem. Phys. Lett., in press.

[21] R. Furrer, J. Gromer, A. Kachar, M. Schwoerer, H.C. Wolf, Chem. Phys. 9, 445 (1975).

[22] H.C. Brenner, J. Chem. Phys. 52, 2328 (1973).

[23] D.D. Anthenius, J. Schmidt, J.H. van der Waals, Mol. Phys. 29, 1521 (1974).

[24] R.H. Clarke, J. Chem. Phys. 52, 2328 (1970).

[25] A.S. Davydov, Theory of Molecular Excitons, Plenum Press, New York 1971. 